\title{
Drowsy Driver Detection System: A Novel Approach Using Haar Like Features
}

\author{
Manoj Kateja ${ }^{1}$, Krunal Panchal ${ }^{2}$ \\ 1, 2 (International Institute of Information Technology, Pune)
}

\begin{abstract}
There are many instances of road accidents round the world due to driver's lack of attention in driving. One of the prime reasons can be drowsiness. In this paper, a drowsiness detection system using Haar like feature technique is implemented for eye detection and a simulator for testing the system was built. An audible alert is connected with the system to acknowledge the drowsiness of the driver. A simulator is made by SDL (Simple Direct Media Layer) which is a cross platform multimedia development API library to provide low level access to joystick, mouse, etc. Whenever the driver is outside the lane in the simulator then also it generates an audible warning signal.
\end{abstract}

Keywords - Eye Detection, Face Detection, Haar like features, USART.

\section{INTRODUCTION}

One of the main reason for the accidents round the world is the lack of attention of the driver. A driver may get distracted by many things. But out of all, the main reason is the drowsiness which may be due to anything like night shifts, heavy workload, etc. There are many methods used to detect a driver's drowsiness. Some of them are: Image processing based techniques such as PERCLOS [1], Haar like features, Physiological signal detection approach using Electroencephalogram (EEG) [2], Electrocardiogram (ECG) [3] and Monitoring the lateral position of the vehicle. This paper presents one such technique for drowsiness detection of a driver.

This paper is divided in following parts: Section II gives the basic idea of the system, Section III presents a detailed approach for the system, Section IV shows the results and the simulator, Section V concludes the paper by its conclusion.

\section{BASIC CONCEPT}

The idea behind the implementation of this system is to use a webcam to detect a driver's eyes. A steering wheel is also interfaced with the PC to drive the car in the simulator. Image processing algorithms are used to detect eye's movement. Serial communication is used to get the acknowledgement of the drowsiness.

A buzzer is interfaced via Atmega8 to get an audible sound alert. SDL library is used to make the simulation for driving conditions.

The basic block diagram is shown in figure 1 .

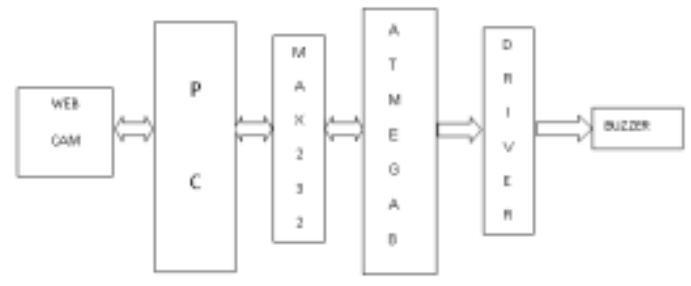

Fig. 1: Block Diagram

III.

APPROACH

A. Smoothening of the captured image

First, an image of the driver is captured via a webcam installed in front of the driver's face. Infrared cameras can be used to minimize illumination problems. An average filter is used to minimize the illumination changes in the image. It is a low pass filter.

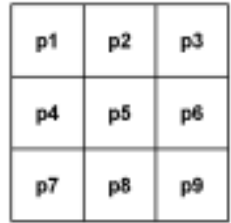

Fig. 2 : $3 * 3$ pixel grid. 
It takes the average of all the neighbouring pixels and compute its sum and then dividing it by the number of pixels in the window. One such window can be seen in Figure 2.

Filtered pixel $(\mathrm{p})=(\mathrm{p} 1+\mathrm{p} 2+\mathrm{p} 3+\mathrm{p} 4 \ldots+\mathrm{p} 9) / 9$

An average filter is used to smooth out the images from camera. The filter used is of $3 * 3$ size. The use of average filter minimises the illumination changes in images. In RGB image format each channel is considered separately for smoothing.

\section{B. Haar like features}

Haar like features was first developed by Viola and Jones [4]. The need for an efficient algorithm for eye detection is very high. Haar like features are used to detect face and eye of the driver. A Haar like feature is a classifier which is trained with a few hundreds of positive and negative examples that are scaled to the same size. Here positive examples refer to the images of an object which is to be detected and negative examples indicate images of anything other than the desired object. A feature based system works faster than a pixel based system. The Haar features are shown in Figure 3.

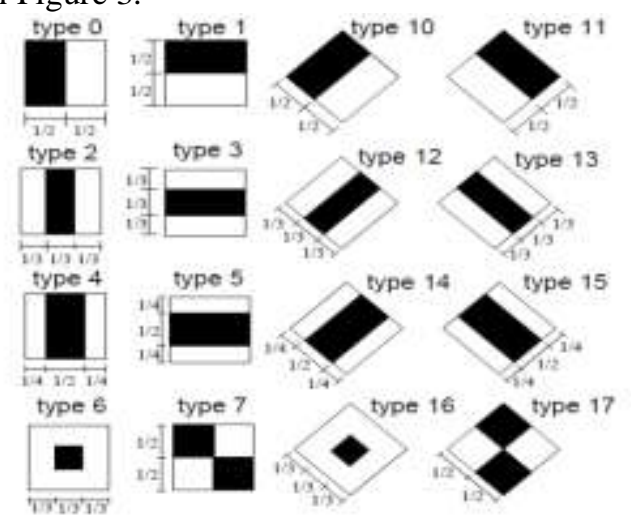

Fig. 3: 18 types of features [4]

There are three main parts in which Haar like features work: Integral Image, Training the classifier and Cascading. An integral image can be obtained by computing the sum of two pixels above and to the left of the original pixel. The idea is to make a rectangle which is used as a feature for the comparison. There are 18 types of features. The rectangle shape is divided into two areas viz. black and white. First take the sum of the black and white individually. Then subtract the sum of white area from the black area.

The task of training the classifier includes the features selected by AdaBoost. AdaBoost is a learning algorithm which uses weak features for classification to form a strong classifier. The weak features are reweighted to correct the previous features.

\section{Haar Cascade Classifiers}

Haar cascade classifier is an algorithm which consists of a group of classifiers providing good detection performance and increases speed of operation. The first classifier evaluates some easy features. A positive result from it will let the second classifier to take place and perform the computation. A negative result will lead to an immediate rejection of the sub-window. The cascade tries to reject the negatives in early stages itself. The detection process is a probabilistic process. The second classifier has a more difficult task than the first one. The cascade classifiers are needed to be trained from the set of detection and performance goals. The detection rate is given by

$$
\mathrm{D}=\prod_{\mathrm{i}=1}^{\mathrm{k}} \mathrm{di}
$$

Where

$\mathrm{D}$ is the detection rate of the classifier; $\mathrm{k}$ is the number of classifiers,

di is the detection rate of the ith classifier [4].

The schematic description of the detection process is shown in Figure 4.

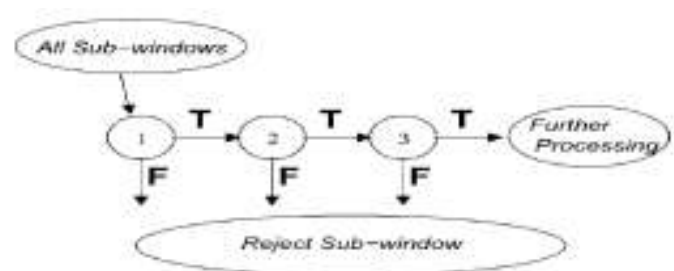

Fig. 4: Schematic description of the detection cascade [4].

$$
\text { www.iosrjournals.org }
$$




\section{Virtual environment simulator}

The virtual environment is developed by using SDL (Simple Direct Media Layer). SDL is a low level cross-platform API library designed to work with joystick, mouse, keyboard, etc. A lane detection algorithm is implemented in the virtual environment. A simulator is made using SDL which has a car in it and can detect the lane. When the car crosses the lane, it generates an audible alert in the form of a buzzer. This environment is used to test the drowsiness detection algorithm [5].

\section{E. Serial Communication}

The use of serial communication becomes essential in this project to interface the buzzer in the system. IC MAX232 is interfaced with the Atmega8 microcontroller for serial communication. It is used to change the voltage levels of PC to microcontroller. The data obtained from the face detection techniques send signal to the buzzer via serial communication. The crystal of $1 \mathrm{MHz}$ is used to give the 4800 baud rate. The value is required to calculate the baud rate. The equation can be given as

$$
\text { UBRR }=\text { Fosc } /(16 * \text { BAUD })-1 \text { [6] }
$$

Where -

Fosc : Input crystal frequency

BAUD: Required baud rate

The schematic diagram for interfacing MAX232 with Atmega8 is shown in Figure 5.

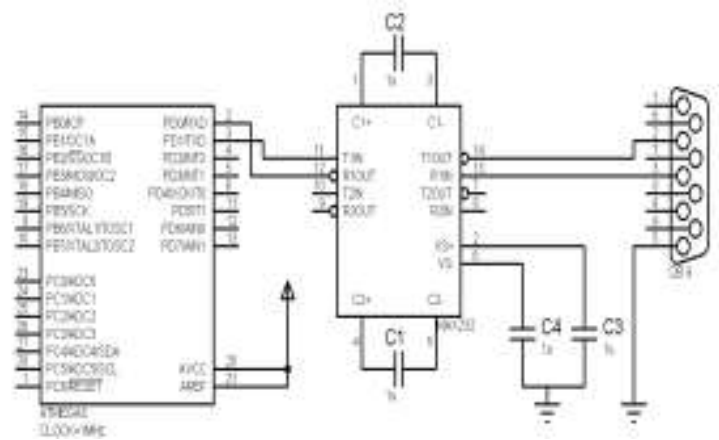

Fig. 5: Interfacing of MAX232 with Atmega8

\section{RESULTS}

The virtual environment of the drowsy driver detection is shown in Figure 6.

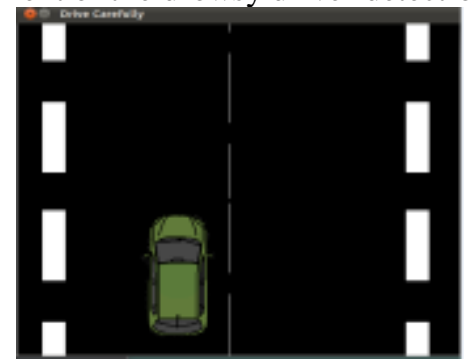

Fig. 6: View of driving simulator

In this simulator, if the car crosses the lane of the road then the system considers it to be a positive response and generates an audible alert. The lane detection algorithm is implemented using OpenCV library which is a computer vision library developed by Intel ${ }^{\circledR}$. The driver's drowsiness can be detected by this when the vehicle go off the road. Figure 7 shows the eye detection of the driver using Haar like features. If the driver's eyes are closed for more than 2 seconds which is set threshold value then the system will generate an alarm signal i.e. buzzer.

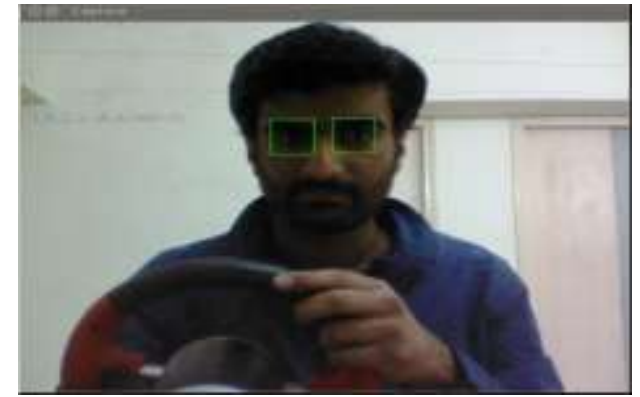

Fig. 7: Driver's eye detection 


\section{V.}

\section{CONCLUSION}

The project uses an image processing technique for face detection i.e. Haar like features to detect the movement of the eyes. SDL is used to make the driving simulator through which the drowsy detection system can be checked. This simulator is tested to get the best results. It can be implemented in a real moving automobile also as a future application.

\section{REFERENCES}

[1] Paul Stephen Rau, "Drowsy Driver Detection and Warning System for Commercial Vehicle Drivers: Field Operational Test Design, Data Analyses and Progress".

[2] Nikhil R Pal, Chien-Yao Chuang, Li-Wei Ko, Chih-Feng Chao, Tzyy- Ping Jung, Sheng-Fu Lieng, Chin-Teng Lin, "EEG-Based Subject- and Session-independent Drowsiness Detection: An Unsupervised Approach".

[3] Gabriela Dorfman Furman, Armanda Baharav, "Investigation of Drowsiness while Driving Utilizing Analysis of Heart Rate Fluctuations".

[4] Paul Viola, Michael J. Jones, "Robust Real-Time Face Detection".

[5] QNX, "Simple Direct Media Layer", [http://www.libsdl.org/].

[6] Datasheets of ATmega8, MAX232. 